\title{
Ordered Bicontinuous Mesoporous Polymeric Semiconductor Photocatalyst
}

\section{Supporting Information}

\author{
Qian Li, Chuanshuang Chen, Chen Li, Ruiyi Liu, Shuai Bi, Pengfei Zhang, \\ Yongfeng Zhou, and Yiyong Mai*
}

School of Chemistry and Chemical Engineering, Frontiers Science Center for Transformative Molecules, Shanghai Key Laboratory of Electrical Insulation and Thermal Ageing, Shanghai Jiao Tong University, 800 Dongchuan Road, Shanghai 200240, China

*E-mail: mai@sjtu.edu.cn

\section{Experimental Section}

\section{Synthesis of $\mathrm{PS}_{213}-b-\mathrm{PEO}_{45}$ block copolymer}

The PS- $b$-PEO block copolymer was prepared by atom transfer radical polymerization (ATRP) method involving two steps (Scheme S1). ${ }^{1}$ In the first step, monomethoxy PEO2000 (50 g, 0.025 mol) was dissolved in freshly distilled dichloromethane (DCM, $200 \mathrm{~mL}$ ) in a flame-dried Schlenk-flask with a stirring bar. After the addition of triethylamine $(10.1 \mathrm{~mL}, 7.5 \mathrm{mmol})$, the mixture was cooled to $0^{\circ} \mathrm{C}$. Then, 2-bromoisobutyryl bromide $(6.2 \mathrm{ml}, 0.05 \mathrm{~mol})$ was added dropwise under stirring. The reaction mixture was stirred for 12 hours in an ice-water bath. Afterwards, the mixture was filtered to remove the triethylamine salt. The residue solution was concentrated by rotary evaporation to ca.50 mL. Then, the resultant solution was added dropwise into $500 \mathrm{~mL}$ cold ether under stirring to precipitate the product. The resulting suspension was stored for 24 hours at ca. $0^{\circ} \mathrm{C}$. The generated white precipitate was filtered and washed with cold ether for three times. The obtained white product was dried under vacuum at room temperature overnight. In the second step, $2 \mathrm{~g} \mathrm{PEO}_{45} \mathrm{-Br}(0.84 \mathrm{mmol}), 133 \mathrm{mg} \mathrm{CuBr}(0.93 \mathrm{mmol})$, and $206 \mu \mathrm{L}$ PMDETA ( $1 \mathrm{mmol}$ ) were added into a dried Schlenk flask. The flask was evacuated and refilled with $\mathrm{N}_{2}$ in three cycles to remove the oxygen. Then, a calculated amount of styrene was transferred to the flask via a syringe. The resulting mixture was further deoxygenated by three freeze-pump-thaw cycles. The flask was then subsequently immersed in a thermostated oil bath at $110{ }^{\circ} \mathrm{C}$ and stirred vigorously. The reaction conversion was monitored by gel permeation 
chromatography (GPC). Upon attainment of the required molecular weight of the designed PS- $b$ PEO sample, the polymerization was terminated by removal of the heat source and subsequently cooling by liquid nitrogen. The gel-like product was dissolved in THF and then passed through a short column of basic alumina to remove copper complexes. Afterwards, the mixture solution was concentrated and then dropped into a large excess of methanol. The resulting white precipitate was filtered and then washed with methanol for at least three times. The final product was dried under vacuum at room temperature for 24 hours.

\section{Characterizations and Measurements}

\section{Transmission electron microscopy (TEM)}

TEM studies were performed using a JEOL JEM-2100 microscope equipped with a LaB6 gun operated at $200 \mathrm{kV}$ (Cs $1.0 \mathrm{~mm}$, point resolution of $2.3 \AA$ ). Images were recorded using a TENGRA CCD camera $(2304 \times 2304$ pixels with a 2:1 fiber-optical taper and an effective pixel size of $18 \mu \mathrm{m}^{2}$ ). TEM sample was prepared by dropping a drop of the sample dispersions onto a copper grid, followed by drying naturally for 24 hours.

\section{Scanning electron microscopy (SEM)}

SEM observations were performed on a JEOL JSM-7401F field emission scanning electron microscope. A low accelerating voltage ( $1 \mathrm{kV}$ with a point resolution of $\sim 1.4 \mathrm{~nm}$ ) was used. SEM sample was prepared by dropping a drop of the sample dispersions onto a silicon wafer, followed by drying naturally for 24 hours. Before dropping the samples, the silicon wafers were cleaned in a bath of $100 \mathrm{~mL} 80 \% \mathrm{H}_{2} \mathrm{SO}_{4}, 35 \mathrm{~mL} \mathrm{H} \mathrm{H}_{2} \mathrm{O}_{2}$ and then $20 \mathrm{~mL}$ Milli-Q water at room temperature. The silicon surface was then dried with compressed nitrogen gas.

\section{Small angle X-ray scattering (SAXS)}

Small-angle X-ray scattering (SAXS) was performed at the beamline X27C, National Synchrotron Light Source (NSLS), Brookhaven National Laboratory (BNL). The wavelength of incident X-ray was $0.1371 \mathrm{~nm}$. Scattering signals were collected by a marCCD 2D detector with a resolution of $79 \mathrm{~mm} /$ pixel. Typical exposure time was between 30 and $120 \mathrm{~s}$. One-dimensional SAXS profiles were obtained by circular averaging of the corresponding two-dimensional scattering patterns 


\section{Nitrogen adsorption-desorption measurement}

Nitrogen adsorption isotherms were measured at 77K on an Autosorb-iQA3200-4 sorption analyzer (Quantatech Co., USA) instrument. Before measurement, samples were degassed in a vacuum at $180{ }^{\circ} \mathrm{C}$ for at least six hours. Brunauer-Emmett-Teller (BET) method was utilized to calculate the specific surface area using adsorption data in a relative pressure range from 0.06 to 0.2. The pore size distributions and pore volumes were derived from the adsorption branches of isotherms using Barrett-Joyner-Halenda (BJH) method.

\section{X-ray diffraction (XRD)}

XRD patterns were recorded on a Rigaku X-ray diffractometer D/MAX-2200/PC at a rate of $6 \%$ min over the range of $5-80^{\circ}(2 \theta)$. The low-angle XRD pattern was recorded on a SmartLab9KW (Rigaku, Japan) spectrometer.

\section{Fourier transform infrared (FTIR) spectroscopy}

FTIR spectra were recorded on a Spectrum 100 (Perkin Elmer, Inc., USA) spectrometer.

\section{X-ray photoelectron spectroscopy (XPS)}

XPS was characterized using a Thermo ESCALAB spectrometer using a Thermo ESCALAB instrument equipped with a monochromated $\mathrm{Al} \mathrm{K} \alpha$ radiation $(1486.6 \mathrm{eV})$. Binding energies were corrected by reference to the $\mathrm{C} 1 \mathrm{~s}$ peak at $284.8 \mathrm{eV}$.

\section{UV-Vis diffuse reflectance spectroscopy (DRS)}

The UV-vis DRS absorption spectra were collected in the wavelength range of 200-800 nm on a UV-vis spectrophotometer (Shimadzu, Japan, UV-2401) with $\mathrm{BaSO}_{4}$ as the reflectance standard.

\section{Photoluminescence spectroscopy (PL)}

Photoluminescence spectra and the corresponding fluorescence decay spectra were recorded via a Princeton Instruments Acton 2500i grating spectrometer.

\section{Nuclear magnetic resonance (NMR)}


${ }^{1} \mathrm{H}$ NMR spectra was recorded using Bruker AVANCEIII 400 spectrometer with $\mathrm{CDCl}_{3}$ as the solvent at $298 \mathrm{~K}$. Tetramethylsilane (TMS) was used as an internal standard.

\section{Gel permeation chromatography (GPC)}

GPC analyses were carried out on a Shimadzu Prominence system with a refractive index detector (Shimadzu RID-10A) at $40{ }^{\circ} \mathrm{C}$, using THF as the eluent at a flow rate of $1 \mathrm{~mL} / \mathrm{min}$ and linear polystyrene as the standard.

Inductively coupled plasma-mass spectroscopy (ICP-MS)

ICP-MS was conducted on a Thermo Fisher instrument (Germany).

\section{Supporting Scheme S1}

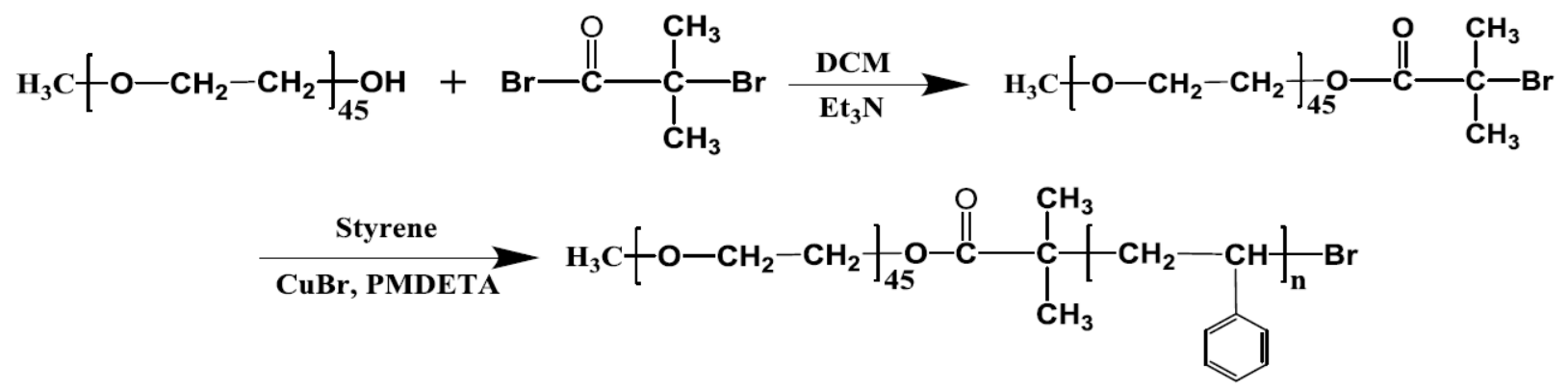

Scheme S1. Synthesis of $\mathrm{PS}_{213}-b-\mathrm{PEO}_{45}$ via ATRP. 


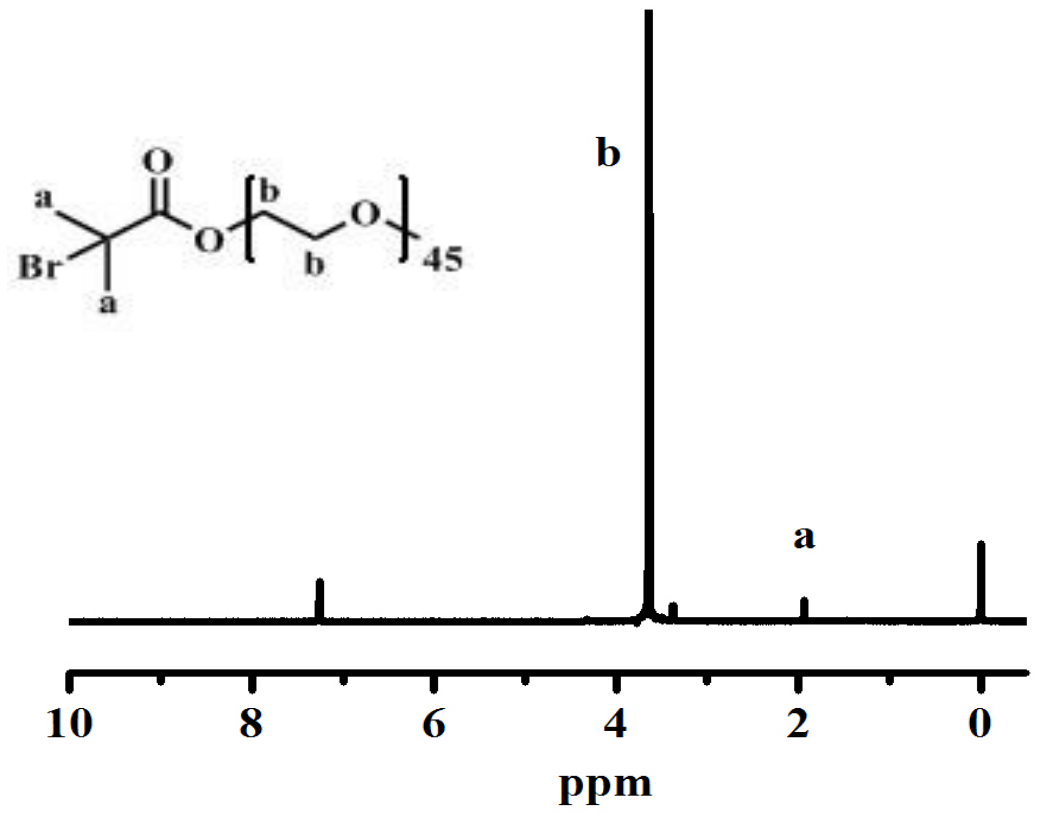

Figure S1. ${ }^{1} \mathrm{H}$ NMR spectrum of $\mathrm{PEO}_{45}-\mathrm{Br}$.

a

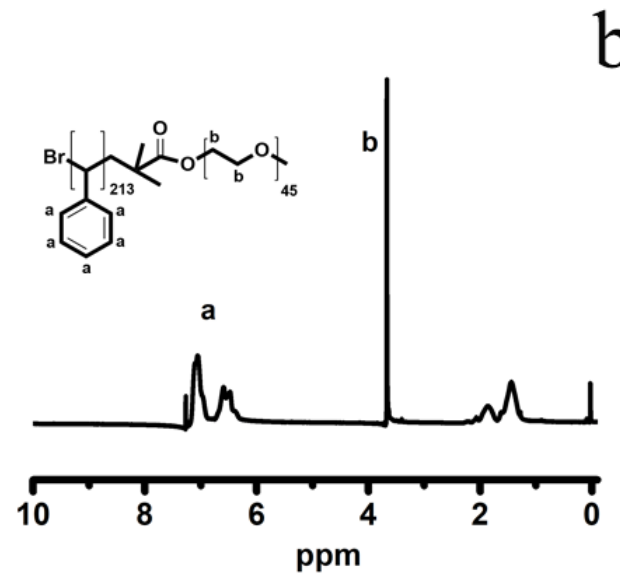

b

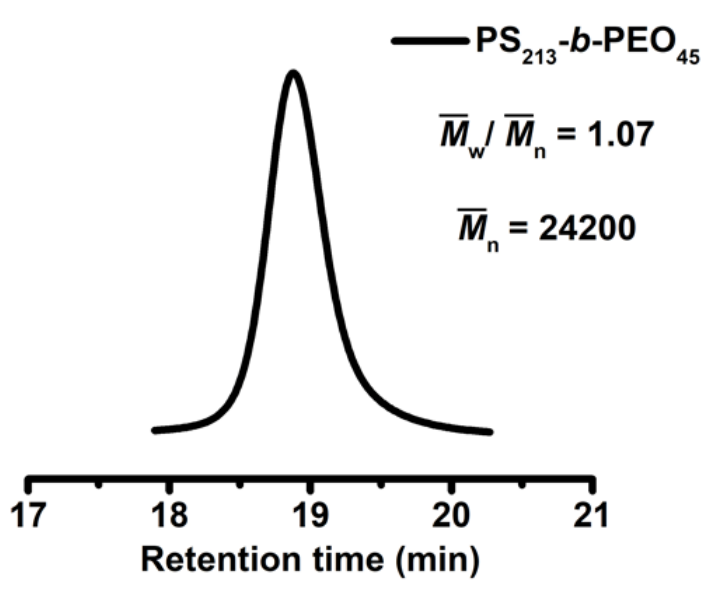

Figure S2. (a) ${ }^{1} \mathrm{H}$ NMR spectrum of $\mathrm{PS}_{213}-b-\mathrm{PEO}_{45}$. (b) GPC trace of $\mathrm{PS}_{213}-b-\mathrm{PEO}_{45}$. The degree of polymerization of the PS block is calculated according to the GPC result, which is close to that obtained from NMR. 


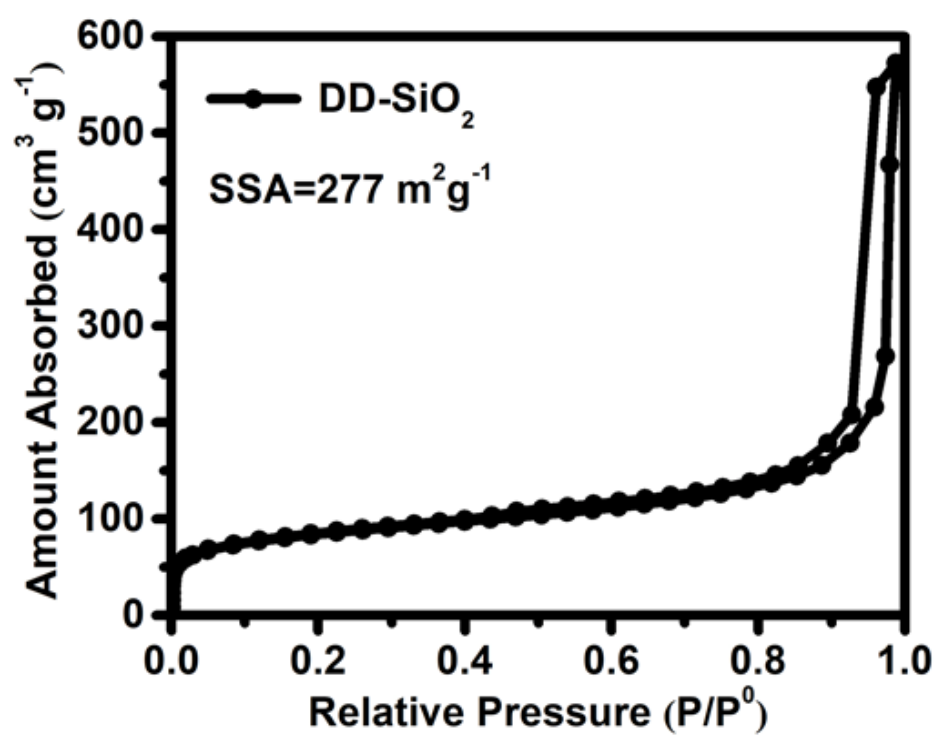

Figure S3. Nitrogen adsorption-desorption isotherm of DD-SiO ${ }_{2}$.
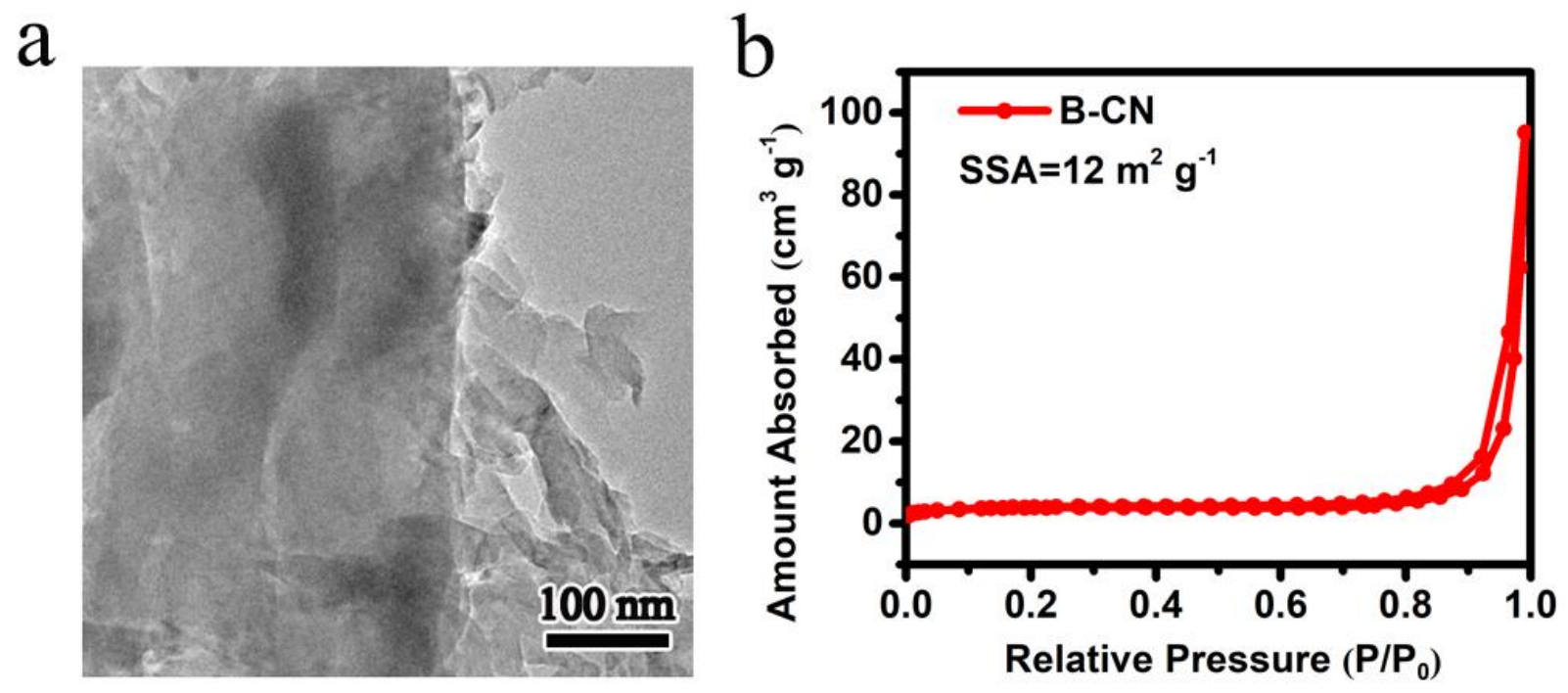

Figure S4. (a) TEM image of B-CN. (b) Nitrogen adsorption-desorption isotherm of B-CN. 

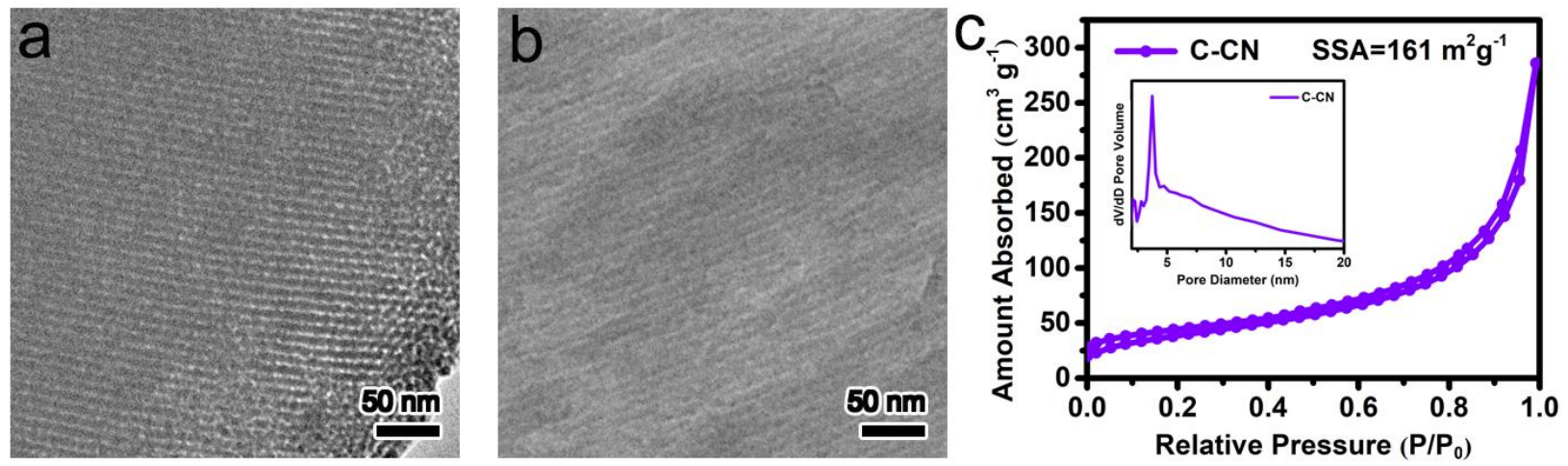

Figure S5. (a) A typical TEM image of silica SBA-15. (b) A typical TEM image of C-CN. (c) Nitrogen adsorption-desorption isotherm of $\mathrm{C}-\mathrm{CN}$; the inset shows the pore size distribution.
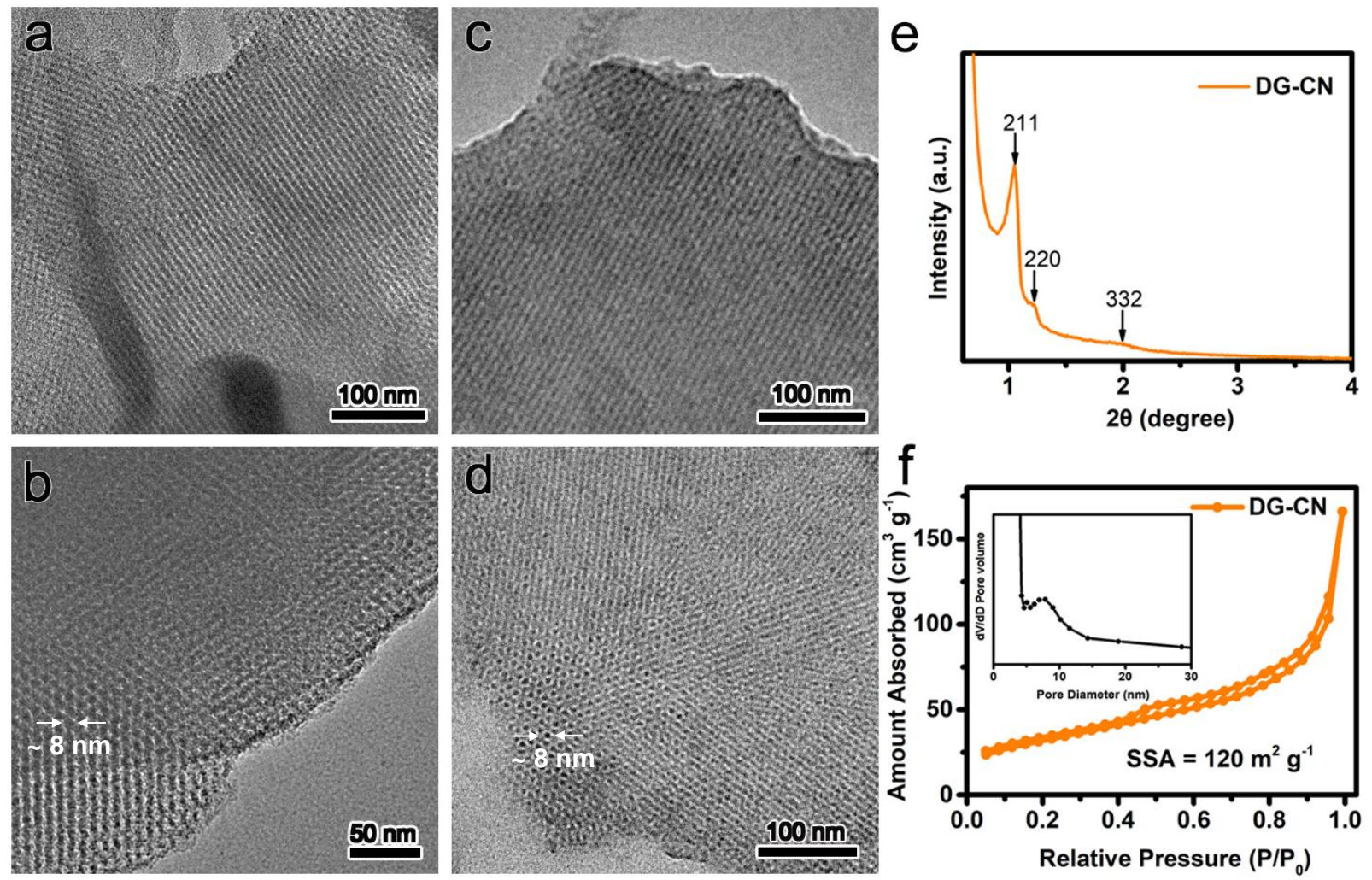

Figure S6. (a,b) TEM images of KIT-6. (c,d) TEM images of DG-CN. (e) Low-angle powder XRD pattern of DG-CN. The diffraction peaks at $2 \theta$ values of $1.05^{\circ}, 1.23^{\circ}$ and $2.01^{\circ}$ can be indexed as the (211), (200) and (332) reflections of a cubic $I a \overline{3} d$ (double gyroid) structure. ${ }^{2,3}$ (f) Nitrogen adsorption-desorption isotherm of DG-CN. 

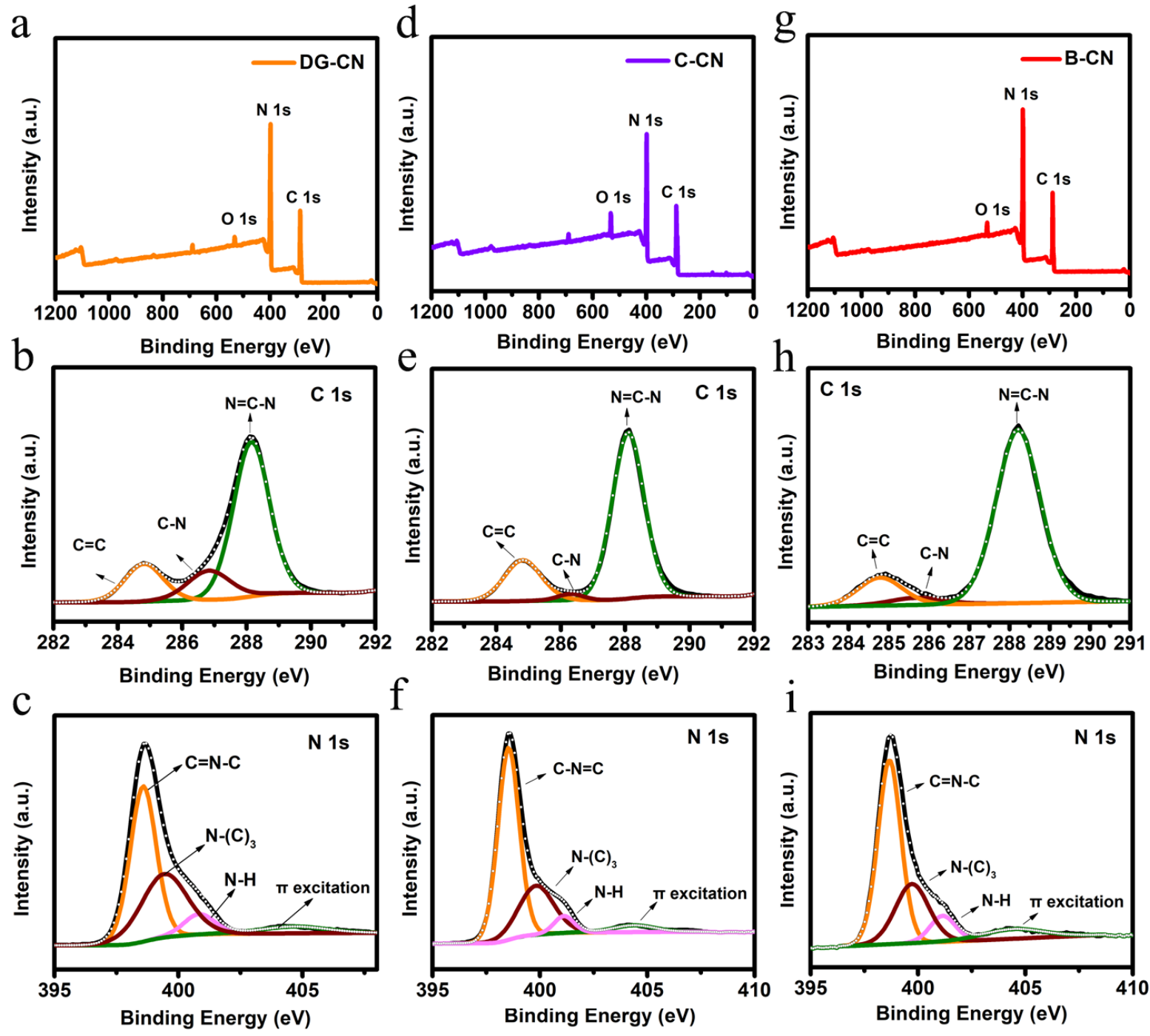

Figure S7. (a) XPS survey spectrum of DG-CN. (b) C 1s and (c) N 1s XPS spectra of DG-CN. (d) XPS full spectrum of C-CN. (e) C 1s and (f) N 1s XPS spectra of C-CN. (g) XPS survey spectrum of B-CN. (h) C 1s and (i) N 1s XPS spectra of B-CN. 


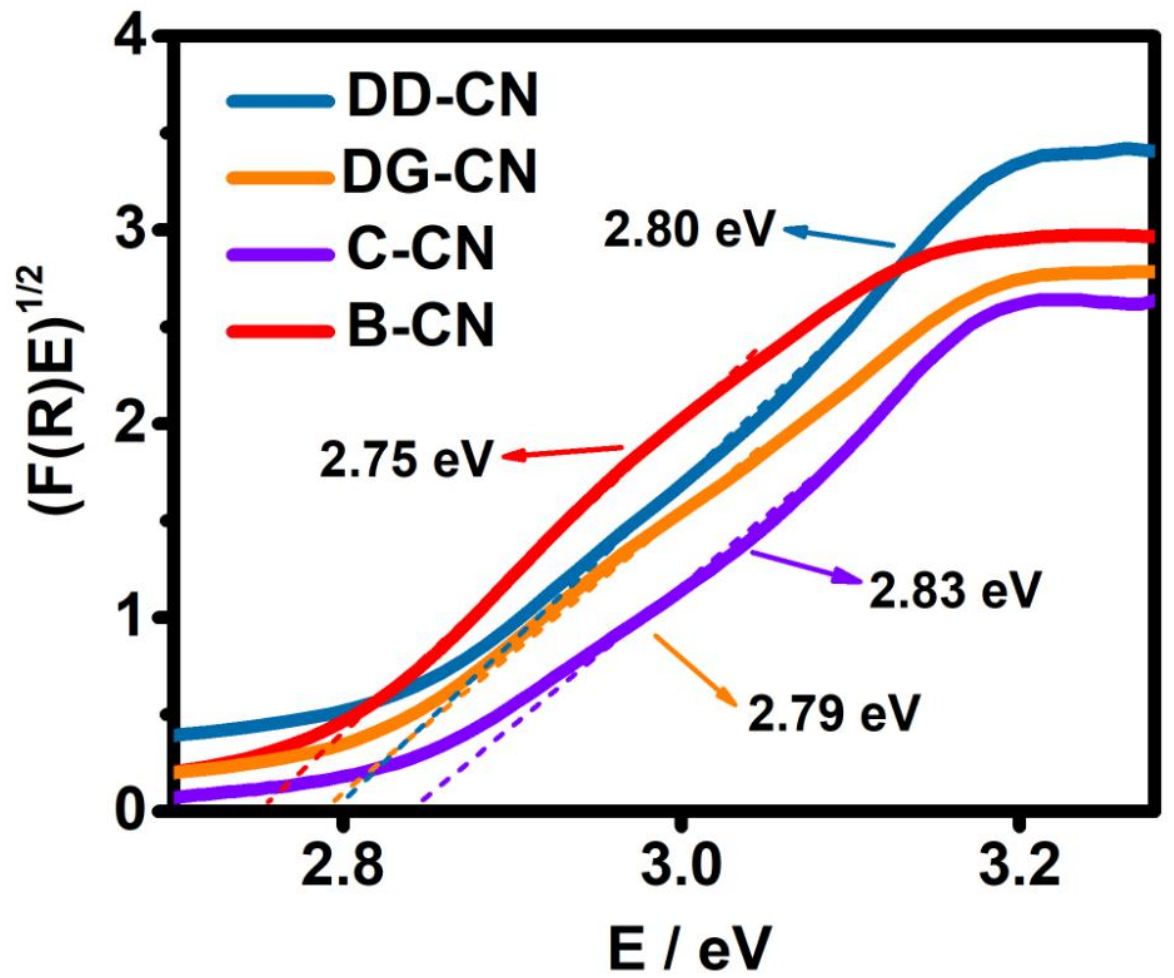

Figure S8. The optical bandgaps of DD-CN, DG-CN, C-CN and B-CN.

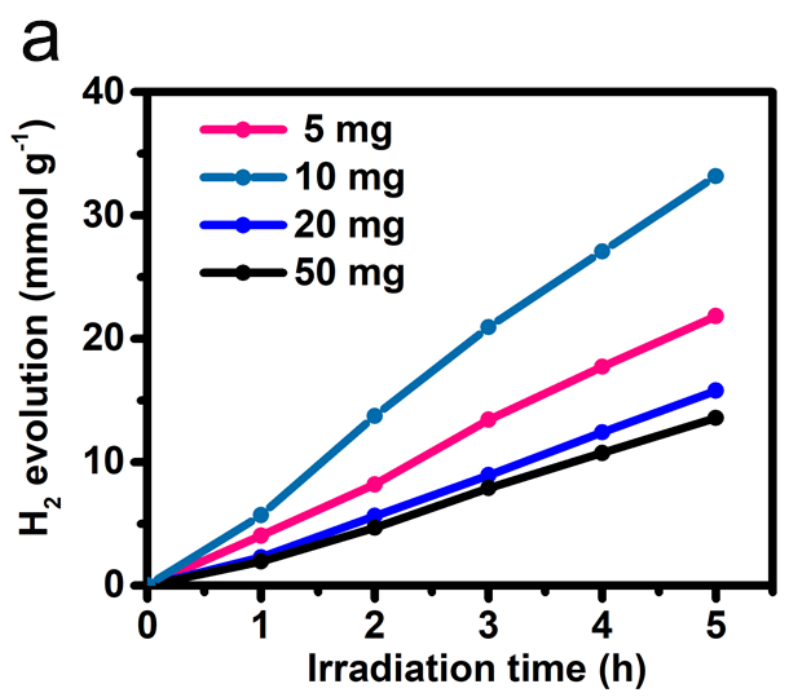

$b$

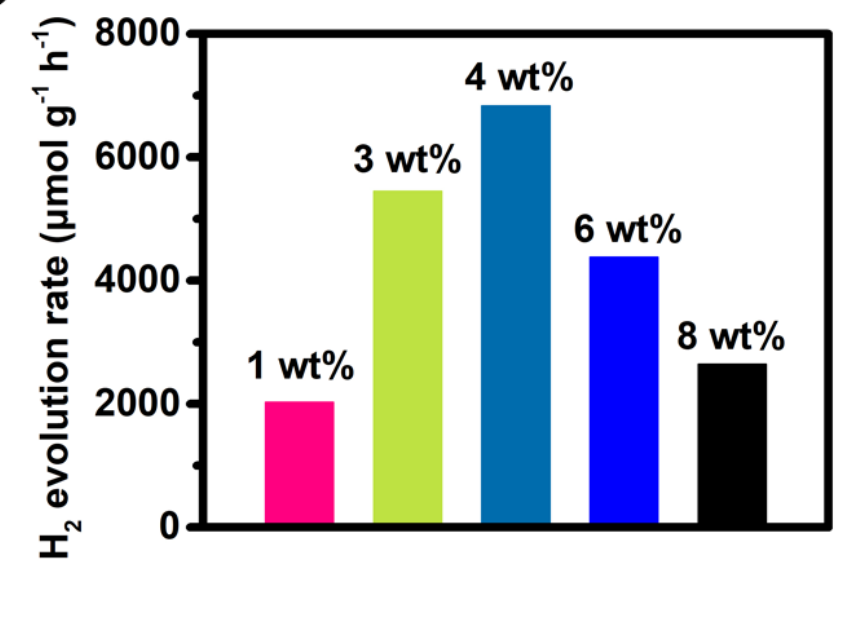

Figure S9. Photocatalytic $\mathrm{H}_{2}$ production rates of the DD-CN samples with different weights (a) or different Pt contents (b) under similar catalytic conditions (for seeking the optimal Pt content, 10 mg DD-CN was employed). 


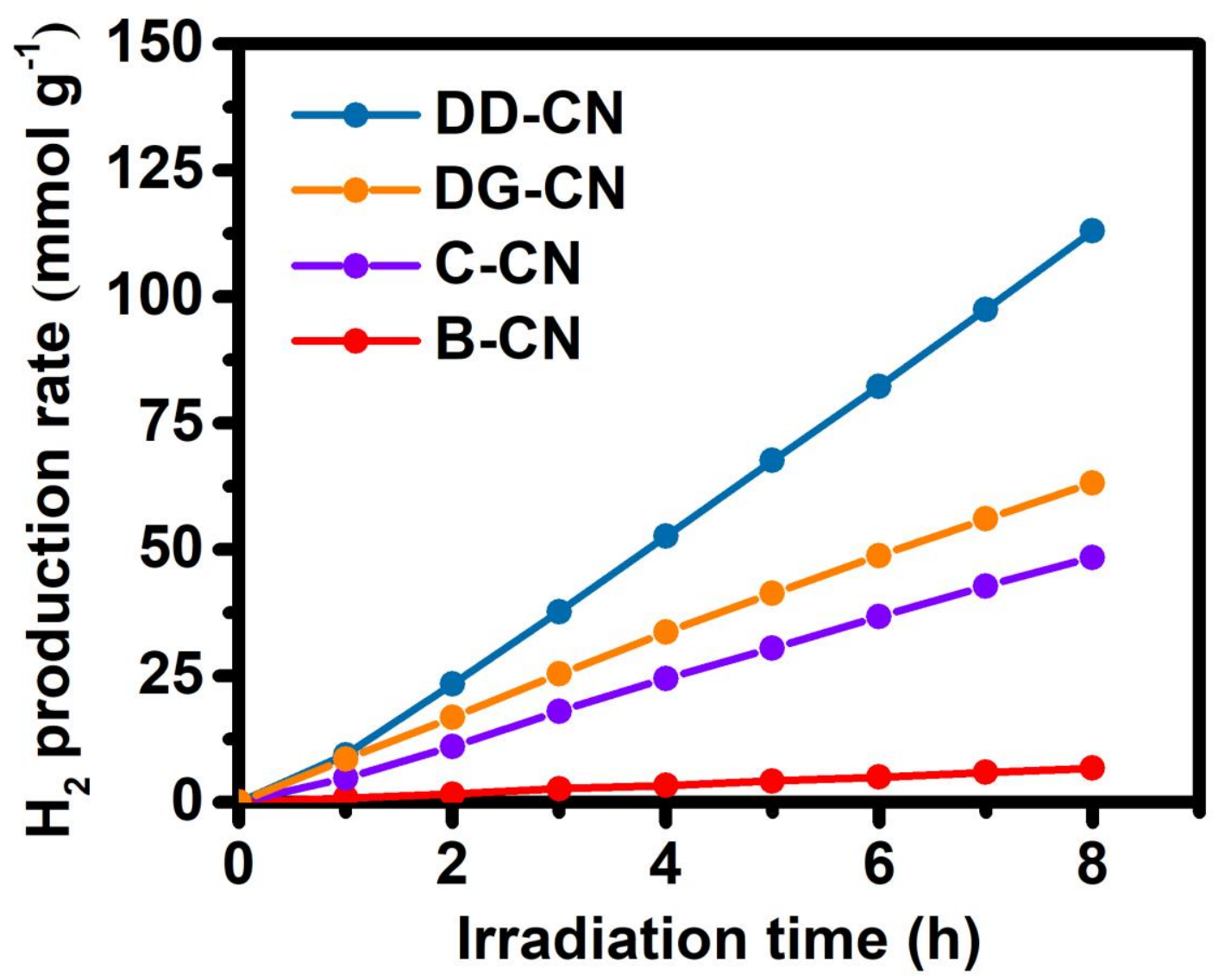

Figure S10. Time-dependent photocatalytic $\mathrm{H}_{2}$ evolution rates of DD-CN (blue), DG-CN (orange), C-CN (purple) and B-CN (red) under $\lambda>300 \mathrm{~nm}$ light irradiation. 

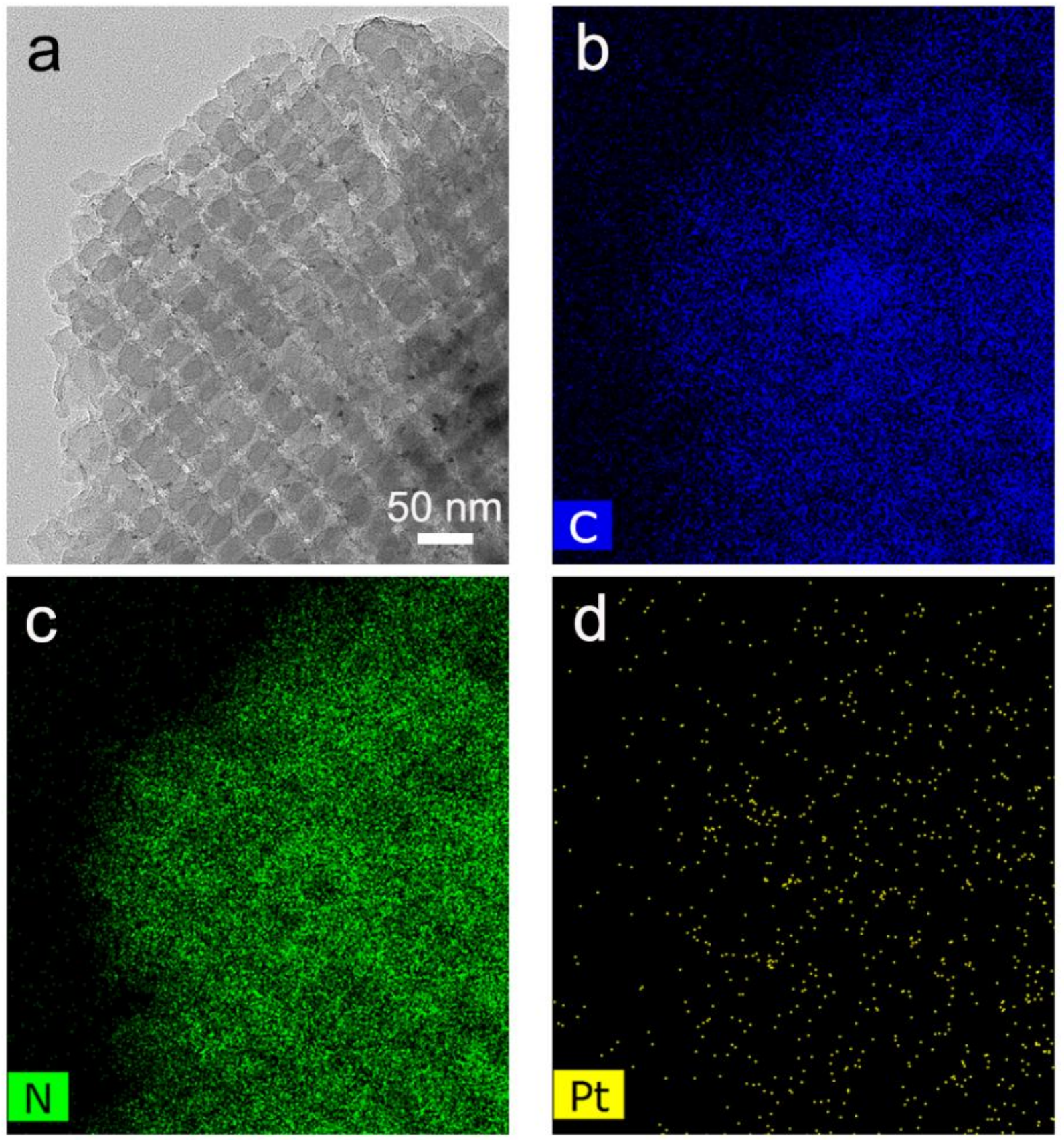

Figure S11. Elemental mapping spectra of the Pt/DD-CN sample with a 4 wt.\% Pt content (an optimum Pt content in our case) after the cycling stability testing. 


\section{Supporting Table S1}

Table S1. The comparison of the photocatalytic $\mathrm{H}_{2}$ production rate of DD-CN with those of some reported porous $\mathrm{CNs}$, evaluated under similar testing conditions.

\begin{tabular}{|c|c|c|c|}
\hline Photocatalyst & $\operatorname{SSA}\left(m^{2} g^{-1}\right)$ & $\begin{array}{l}\mathrm{H}_{2} \text { production rate } \\
\left(\mu \mathrm{mol} \cdot \mathrm{g}^{-1} \cdot \mathrm{h}^{-1}\right)\end{array}$ & Ref. \\
\hline DD-CN & 131 & 6831 & This work \\
\hline $\begin{array}{l}\text { Porous few-layer carbon } \\
\text { nitride }\end{array}$ & 164 & 7990 & $\begin{array}{l}\text { J. Am. Chem. Soc. 2019, } \\
\text { 141, 2508-2515. }\end{array}$ \\
\hline Cubic mesoporous g-CN & 197 & 5250 & $\begin{array}{l}\text { J. Mater. Chem. A 2017, 5, } \\
\text { 16179-16188. }\end{array}$ \\
\hline $\begin{array}{l}\text { 3D ordered close-packed } \\
\text { g-CN nanosphere }\end{array}$ & 170 & 3137 & $\begin{array}{l}\text { Angew. Chem. Int. Ed. 2019, } \\
\text { 58, 4587-4591. }\end{array}$ \\
\hline $\begin{array}{l}\text { Ordered mesoporous g- } \\
\mathrm{C}_{3} \mathrm{~N}_{5}\end{array}$ & 297 & 2670 & $\begin{array}{l}\text { Angew. Chem. Int. Ed. 2017, } \\
56,8481-8485 \text {. }\end{array}$ \\
\hline Mesoporous g-CN & 109 & 1917 & $\begin{array}{l}\text { Appl. Catal., B 2018, 232, } \\
384-390 .\end{array}$ \\
\hline $\begin{array}{l}\text { Macro-/mesoporous g- } \\
\text { CN }\end{array}$ & 219 & 1900 & $\begin{array}{l}\text { Nano Energy 2018, 50, 376- } \\
382 .\end{array}$ \\
\hline $\begin{array}{l}\text { Macro-/mesoporous P- } \\
\text { doped g-CN nanosheet }\end{array}$ & 123 & 1596 & $\begin{array}{l}\text { Energy Environ. Sci., 2015, } \\
\text { 8, 3708-3717. }\end{array}$ \\
\hline Carbon nitride aerogel & 133 & 600 & $\begin{array}{l}\text { Angew. Chem. Int. Ed. 2017, } \\
129,11045-11050 .\end{array}$ \\
\hline $\begin{array}{l}\text { Carbon Quantum dot- } \\
\text { doped g-CN }\end{array}$ & 120 & 3538 & $\begin{array}{l}\text { Angew. Chem. Int. Ed. 2018, } \\
130,5867-5873 .\end{array}$ \\
\hline $\begin{array}{l}\text { Single Ag atom-doped } \\
\text { g-CN }\end{array}$ & 157 & 790 & $\begin{array}{l}\text { ACS Nano 2016, 10, 3166- } \\
3175 \text {. }\end{array}$ \\
\hline
\end{tabular}




\section{Supporting References}

(1). Mai, Y.; Eisenberg, A. Controlled Incorporation of Particles into the Central Portion of Vesicle Walls. J. Am. Chem. Soc. 2010, 132, 10078-10084.

(2). Kim, T-W.; Kleitz, F.; Paul, B.; Ryoo, R. MCM-48-Like Large Mesoporous Silicas with Tailored Pore Structure: Facile Synthesis Domain in a Ternary Triblock Copolymer-ButanolWater System. J. Am. Chem. Soc. 2005, 127, 7601-7610.

(3) Liu, B.; Yang, M.; Xia, N.; Zheng, P.; Wang, W.; Burger, C. Gyroid Nanostructure through Manipulation of Unique Molecular Shape, Polarity and Functionalization of a Janus Amphiphilic Codendrimer. Soft Matter 2012, 8, 9545-9552. 